\title{
Screening and Analysis of COVID-19 cases in non- epidemic areas: A Retrospective Study
}

\section{Yan Zhu}

Department of Infectious Diseases, Southwest Hospital, Third Military Medical University (Army Medial University) https://orcid.org/0000-0002-8925-3228

\section{Yan Guo}

Department of Infectious Diseases, Southwest Hospital, Third Military Medical University (Army Medial University) https://orcid.org/0000-0002-7072-5344

\section{Juan Ma}

Department of Pediatrics, North-KuanRen General Hospital https://orcid.org/0000-0002-2530-2043

\section{Mao-shi Li}

Department of Infectious Diseases, Southwest Hospital https://orcid.org/0000-0002-0316-169X

\section{Jia-fei Chen}

Department of Radiology, Southwest Hospital https://orcid.org/0000-0002-2511-4798

\section{Ming Liu}

Department of Infectious Diseases, Southwest Hospital, Third Military Medical University (Army Medial University) https://orcid.org/0000-0002-5244-2973

\section{Ze-hui Yan}

Department of Infectious Diseases, Southwest Hospital, Third Military Medical University (Army Medial University) https://orcid.org/0000-0002-8887-812X

\section{Guo-hong Deng}

Department of Infectious Diseases, Southwest Hospital, Third Military Medical University (Army Medial University) https://orcid.org/0000-0003-1263-7220

\section{Qing Mao ( $\sim$ QingMao@tmmu.edu.cn )}

Department of Infectious Diseases, Southwest Hospital, Third Military Medical University (Army Medial University) https://orcid.org/0000-0003-3138-2104

Hui-min Liu ( $\square$ liuhuimin_412@sina.cn )

Department of Infectious Diseases, Southwest Hospital, Third Military Medical University (Army Medial University) https://orcid.org/0000-0003-4912-2001

\section{Research Article}

Keywords: screening; COVID-19; suspected standard; a retrospective study; Chongqing

Posted Date: June 2nd, 2020 
DOI: https://doi.org/10.21203/rs.3.rs-32811/v1

License: (c) (1) This work is licensed under a Creative Commons Attribution 4.0 International License. Read Full License 


\section{Abstract}

Objective: To compare the epidemiological and clinical characteristics of confirmed and suspected corona virus disease 2019 (COVID-19) cases via the process of "triage-screening-isolation-transfer" in the hospitals of non-epidemic areas.

Methods: The general data, epidemiological history, clinical symptoms, laboratory examination, and chest computed tomography (CT) imaging characteristics of 38 patients with suspected COVID-19, admitted between January 21 and March 5, 2020, were analyzed.

Results: According to the results of the novel severe acute respiratory syndrome coronavirus (SARS-CoV2) ribonucleic acid (RNA) testing, the patients were divided into study group (RNA positive) and control group (RNA negative). Ultimately, 8 cases were RNA-positive and diagnosed as CDVID-19, and 30 cases were negative. Approximately half of the patients in the study group returned to Chongqing from Wuhan; this number was significantly larger than that of the control group $(P<0.05)$. The number of subjects in close contact with the confirmed cases with SARS-CoV-2 RNA-positive and the incidence of aggregation was significantly larger in the study group than in the control group (both $\mathrm{P}<0.05$ ). The clinical symptom of the study group was mainly low fever (with or without cough). The patients with decreased white blood cells (WBC) in the study group were significantly more than those in the control group $(P<0.05)$. Both group had reduced lymphocytes (Lym) but the number of patients with increased C-reactive protein (CRP) in the study group was significantly more than that in the control group $(P<0.05)$. There were different degrees of chest CT abnormalities in both study and control group $(P>0.05)$.

Conclusion: The epidemiological investigations in screening for infectious diseases is crucial. The risk of infection was high from the primary epidemic area and/or in close contact with the confirmed case. The most common form of clustering occurrence was family aggregation. CDVID-19 was mainly characterized by fever and respiratory symptoms, although asymptomatic infection may also occur. Decreased WBC, decreased Lym, and increased CRP are common characteristics but can also be combined with other respiratory tract virus infections. COVID 19 screening by chest CT alone had certain limitations in non- epidemic areas.

\section{Background}

In late December 2019, a mass outbreak of unexplained pneumonia was reported in Wuhan, China (1), which has now become a global epidemic. As of March 21, 2020, comprehensive data from the National Health Commission, the Center for Disease Control (CDC), and the World Health Organization (WHO) were released, showing a total of 81,456 confirmed cases and 3,261 deaths in China. Globally, there have been 275,429 confirmed cases and 11,397 deaths. On January 7,2020 , China quickly identified the pathogen as a novel coronavirus ${ }^{(2)}$. On January 12 , WHO officially named it as "2019 novel coronavirus (2019nCoV)" (3). On February 8, the Chinese National Health Commission named the disease caused by this virus as novel coronavirus pneumonia (NCP). On February 11, WHO changed the name of the disease to 
COVID-19. Consecutively, the Coronaviridae Study Group of the International Committee on Taxonomy of Viruses renamed the virus as SARS-CoV-2, as it was homologous to the 2003 SARS coronavirus ${ }^{(2-5)}$. SARS-CoV-2 has been confirmed to propagate by human-to-human transmission pattern, and the basic reproduction number (R0) is estimated to be 2.2 according to the early epidemiological survey, which is greater than that of SARS and Middle East respiratory syndrome (MERS) ${ }^{(6-9)}$. The average incubation period of COVID-19 is 5-7 days and 14 days is considered as the appropriate medical observation period $(6,10,13)$.

\section{Introduction}

Some researchers ${ }^{(11)}$ established a model based on the comprehensive factors, such as the geographical location and traffic flow of Wuhan, and predicted that Chongqing, with a population of 32 million, could become the next city with the outbreak of the epidemic after Wuhan if not controlled in time. By March 21, 2020, a total of 576 cases had been confirmed; of these, 570 had been cured and 6 were deceased.

However, from February 25 to March 21, 2020, no new cases were reported in Chongqing. Recently, all the confirmed cases treated in the hospitals of the city have been cleared. Therefore Chongqing did not become the next Wuhan, there is a long-awaited turning point. Controlling the source of infection, cutting off the transmission route, and protecting the susceptible people are three important links in the prevention and control of the epidemic. The Chinese government has taken a series of effective measures, and front-line medical staff and scientists have played a leading role in responding to the outbreak of COVID-19.

The outbreak in China is now under control, but the number of cases worldwide is increasing rapidly. After the SARS outbreak in 2003, China has abundant experience in setting up fever clinics and establishing outpatient triage strategy, which greatly improves the effectiveness of cutting off the transmission channels ${ }^{(12)}$. According to the requirements of the overall epidemic planning and the geographical characteristics, Chongqing had set up four local COVID-19 designated hospitals, while the other nondesignated hospitals undertook the screening of suspected cases. From January 21 to March 5, 2020, 1087 cases of fever were received in the fever clinic of Chongqing Southwest Hospital, and 38 cases with high suspicion of COVID-19 were screened for observation. By analyzing and summarizing the general data, clinical symptoms, epidemiology, laboratory examination, imaging, diagnosis, and subsequent treatment of the 38 cases, the present study sorts out the process of "triage-screening-isolation-transport" (Fig. 1) and provides experience for the screening of cases in non-epidemic areas.

\section{Materials And Methods}

\subsection{General data}

This retrospective study analyzed the data of 38 cases, consisting of equal number of males and females, suspected with COVID-19 diagnostic criteria. The age of the youngest patient was 3 years, and 
that of the oldest was 71 years (average: $39.1 \pm 14.4$-years-old). According to the results of SARS-CoV-2 RNA testing, the patients were divided into study group (RNA positive) and control group (RNA negative).

\subsection{Screening process (Fig. 1)}

Hospital entrance: Infrared temperature scanner is set up, the distance between people is $>1 \mathrm{~m}$ with appropriate protection to reduce the risk of cross-infection. Cases with fever and/or respiratory symptoms were diverted to the fever clinic.

Fever clinic: Step 1: epidemiological investigation, measurement of body temperature, measurement of oxygen saturation; Step 2: Based on step 1, it is determined whether blood routine and/or chest CT needs to be conducted; Step 3: Based on Steps 1 and 2, it is determined whether SARS-CoV-2 RNA should be tested with fluorescent RT-PCT. While waiting for RNA results, the patients who fulfilled the suspected diagnostic criteria of COVID-19 were isolated and treated.

\subsection{Diagnostic criteria}

According to the definitions of WHO (https://www.who.int/), CDC (https://www.cdc.gov/), NICE (National Institute of Health and Clinical Research) award for excellence (https://www.nice.org.uk/), National Health Commission, PRC (http://www.nhc.gov.cn/), and National Administration of Traditional Chinese Medicine (http://www.satcm.gov.cn/) ${ }^{(13)}$.

\subsubsection{Suspected standard}

The diagnosis of suspected COVID-19 cases should meet the following criteria: (1) three clinical criteria; (2) two clinical standards and one epidemiological standard.

Clinical criteria: (1) fever and/or cough, sore throat, and other respiratory symptoms; (2) Imaging evidence of pneumonia or acute respiratory distress syndrome; (3) reduced or normal white blood cell count or reduced Lym count.

Epidemiological criteria: (1) stay or travel in Wuhan or other communities with reported cases within 14 days prior to the onset of symptoms; (2) expose to SARS-CoV-2-infected case (RNA positive) within 14 days before the onset of symptoms;(3) contact with fever and/or respiratory symptoms in Wuhan or other communities with reported cases within 14 days before the onset of symptoms; (4) there are clustered cases of the disease.

\subsubsection{Diagnostic criteria}

Based on the suspected cases, real-time reverse transcription polymerase chain reaction (RT-PCR) was used to detect the presence of SARS-CoV-2 in the case samples. Also, next-generation gene sequencing was applied to confirm the same ${ }^{(2)}$. The following primers and probe targeted to the envelope gene of SARSCoV2 were used: forward primer 5'-ACTTCTTTTTCTTGCTTTCGTGGT-3'; Reverse primer 5'- 
GCAGCAGTACGCACACAATC-3'; Probe 5'CY5-CTAGTTACACTAGCCATCCTTACTGC-3'BHQ1. The conditions of amplification were as follows: $50{ }^{\circ} \mathrm{C}$ for $15 \mathrm{~min}, 95^{\circ} \mathrm{C}$ for $3 \mathrm{~min}$, followed by 45 cycles of 95 ${ }^{\circ} \mathrm{C}$ for $15 \mathrm{~s}$ and $60{ }^{\circ} \mathrm{C}$ for $30 \mathrm{~s}{ }^{(5)}$.

\subsection{Observation indicators}

General data (gender, age), epidemiological investigation (the history of living or traveling in the epidemic area, contact history, activity tracking, time of onset, length of stay in hospital), clinical symptoms, laboratory examination on admission (CRP, WBC, Lym, neutrophils (Neu), alanine aminotransferase (ALT), creatinine (Cr), glucose (Glu), lactate dehydrogenase (LDH) , procalcitonin (PCT), detection of influenza, avian influenza, respiratory syncytial virus, adenovirus, parainfluenza virus, Epstein-Barr virus (EBV) in the respiratory specimens), and imaging examination chest CT).

\subsection{Statistical methods}

SPSS22.0 statistical software was used to analyze the measurement data with the positive distribution. T or $T^{\prime}$ test was used to express the measurement data with mean \pm standard deviation $(\bar{\chi} \pm S D)$. The measurement data with uneven variances were measured by the rank-sum test, and expressed by the median and quartile spacing $M(Q 25, Q 75) \cdot \chi^{2}$ test was used to express the measurement data with the rate $(\%) . P<0.05$ indicated statistical significance.

\subsection{Subsequent treatment}

According to the diagnosis and treatment guidelines of COVID-19 (13), if RT-PCR was positive, COVID-19 was confirmed. According to the condition, $50 \mu \mathrm{g}$ of recombinant interferon $\mathrm{a} 1 \mathrm{~b}$ atomized and lopinavir and tonavir tablets (Aluvia) 500 mg were administered orally, twice daily, and the patient was prepared for transfer to the designated hospital. If RT-PCR was negative, it was performed again at an interval of more than $24 \mathrm{~h}$ on throat swab, saliva or sputum, and anal swab samples. Patients with more than two times negative RT-PCR results could be discharged. According to the situation, isolation at home for more than 14 days, or transfer to the corresponding department for the treatment of other diseases was recommended.

\section{Results}

\subsection{General data}

Of the 38 screened cases, 8 (average age, $43.25 \pm 10.6$ years) were in the study group, and 30 (average age $38.03 \pm 15.26$ years) constituted the control group $(P>0.05$, no significant difference was detected in the age). In the study group comprised of $37.5 \%$ (3/8) males and $62.5 \%(5 / 8)$ Females, while the control group consisted of $53.3 \%$ (16/30) males and $46.7 \%(14 / 30)$ females $(P>0.05)$. The overall onset time of the symptoms was $3.5(1.8-5.3)$ days. Onset time was $5.0(0.0-5.0)$ days in the study group and 3.0 (2.0-6.3) days in the control group $(P>0.05)$. The overall length of stay in the hospital was 3.0 (3.0-3.3) 
days. The length of stay was $3.0(3.0-3.0)$ days in the study group and $3.0(0.3-5.0)$ days in the control group $(P<0.05)$.

\subsection{Epidemiological situation}

Among the screened cases, $21.1 \%$ (8/38) returned to Chongqing from Wuhan. Of these, $50.0 \%(4 / 8)$ comprised the study group, and the remaining $23.3 \%$ (4/30) formed the control group. $10.0 \%(3 / 30)$ of the control group returned to Chongqing from other cities of Hubei outside Wuhan $(P<0.05) .15 .8 \%$ $(6 / 38)$ of the cohort had close contact with confirmed cases tested positive for RNA of SARS-CoV-2, while $26.3 \%(10 / 38)$ had brief contact. The study group had $50.0 \%$ (4/8) close contact and $12.5 \%(1 / 8)$ brief contact. The control group had 6.7\% (2/30) close contact and 30.0\% (9/30) short contact. However, the subset of patients with close contact in the study group was significantly higher than that in the control group $(P<0.05)$. Conversely, the proportion of temporary exposure in the study group was significantly smaller than that in the control group $(P<0.05) .31 .6 \%(12 / 38)$ of the cases were clustered, including study group $87.5 \%(7 / 8)$ and control group $6.7 \%(5 / 30)$; the study group was significantly larger than the control group $(P<0.05)$. On the other hand, $10.5 \%(4 / 38)$ came in contact with suspected cases, accounting for $13.3 \%(4 / 30)$ in the control group, which had no significant difference compared to the study group $(P>0.05)$.

\subsection{Mainly clinical symptoms}

Among the 38 cases, $42 \%$ (16/38) had fever (with or without cough). Among them, $87.5 \%(14 / 16)$ had a low fever, and $15 \%(2 / 16)$ had a body temperature higher than $38^{\circ} \mathrm{C}$. In the study group, $37.5 \%(3 / 8)$ had fever (with or without cough), $25.0 \%$ (2/8) were asymptomatic, $25.0 \%(2 / 8)$ presented fatigue, muscle soreness, and headache, and $12.5 \%$ (1/8) experienced abdominal discomfort and diarrhea. In the control group, $43.3 \%$ (13/30) had fever (with or without cough), 36.7\% (11/30) had cough (without fever), 10\% $(3 / 30)$ had sore throat and itchy throat, while fatigue, muscle ache, headache, dyspnea, and asymptomatic condition accounted for 3.3\% (1/30) each, respectively. Compared to the study group, the rate of cough (without fever) in the control group was significantly higher $(P<0.05)$.

\subsection{Laboratory inspection}

\subsubsection{Biochemical indexes}

Any statistically significant differences were not detected in the absolute values of WBC, Lym, Neu, ALT, $\mathrm{Cr}$, Glu, LDH, CRP, and PCT between the study and control groups $(P>0.05)$. The number of patients with decreased WBC in the study group was significantly more than that in the control group (37.5\% vs. $0.0 \%$, $P<0.05)$. Both the study and control groups showed reduced Lym $(50.0 \%$ vs. $40.0 \%, P>0.05)$. The number of patients with increased CRP in the study group was significantly more than that in the control group $(87.5 \%$ vs. $46.7 \%, \mathrm{P}<0.05)$.

\subsubsection{Respiratory virus}


Among the 38 cases, 8.4\% (7/38) were infected with influenza A, 5.3\% (2/38) with influenza B, 13.2\% $(5 / 38)$ were infected with both influenza $A$ and $B$, and there was 1 case of respiratory syncytial virus, 1 case of adenovirus, and 1 case of EBV, accounting for $2.6 \%$ each, respectively. $12.5 \%(1 / 8)$ of the study group was infected with influenza B, while $12.5 \%(1 / 8)$ experienced a combination of influenza $A$ and $B$ infection. In the control group, 23.3\% (7/30) was infected with influenza $A, 13.3 \%(4 / 30)$ was infected with influenza $A$ and influenza $B$, and $3.3 \%(1 / 30)$ was infected with influenza $B$ virus, respiratory syncytial virus, adenovirus, and EBV $(P>0.05)$.

\subsection{Imaging features}

Among the 38 cases of chest CT, no significant difference was detected between the study and control groups $(P>0.05)$. In the study group, $62.5 \%(5 / 8)$ of chest CT presented bilateral diffuse or multifocal lesions; $25.0 \%(2 / 8)$ in the left lower lung and $12.5 \%(1 / 8)$ in the double lower lung. The lesions in all 8 cases were characterized by ground glass opacity $(P>0.05)$.

\subsection{Diagnosis}

Among the 38 cases, 8 were confirmed and 30 were excluded. No missed diagnosis was known in the 14day follow-up.

\section{Discussion}

SARS-CoV-2 belongs to coronaviridae, which is an enveloped virus with a non-segmented, positive sense RNA. SARS-CoV-2 is the coronavirus that caused another outbreak after SARS-CoV in 2003 and MERSCoV in 2012. SARS-CoV and MERS-CoV have a high fatality rate of $10 \%$ and $37 \%$, respectively $(14,15)$. Hitherto, the mortality rate of COVID-19 is lower than that of SARS or MERS. However, SARS-CoV-2 is highly infectious, which has become a public health concern as it is threatening to human health and safety.

In this study, 38 suspected cases of COVID-19 were observed in our hospital, with equal proportion of males and females. The average age of the cohort was in the middle-age group, which was considered to be related to the frequency of outgoing activities. The onset time of confirmed cases was about 5 days, which was consistent with the early reports of Wuhan ${ }^{(5)}$ stating the duration as $4.1-7.1$ days. The suspected cases need to be kept in the hospital for observation for about 3 days to complete COVID-19related tests, which included at least two times RT-PCR detection (the interval time should be $>24 \mathrm{~h}$ ) and the time spent in the RT-PCR detection process (approximately $4 \mathrm{~h}$ ). In the clinical setting, the estimated days for observation can be informed in advance, which can soothe the emotions and reduce the occurrence of common negative emotions, such as anxiety. In this study, the diagnostic rate of cases returning from Wuhan to Chongqing was markedly high. Thus, we need to focus on the dynamic changes of the epidemic situation and on the personnel returning from the epidemic area. The risk of COVID-19 was significantly higher in patients who had close contact with confirmed cases with positive SARS-CoV2 RNA. The 12 cases of clustering occurrence were in 4 families, of which, 3 were eventually diagnosed 
positive. This situation supports the human-to-human transmission mode of SARS-CoV-2 as reported previously ${ }^{(7)}$. In a French epidemiological study of COVID-19 cases ${ }^{(16)}$, the risk of exposure was divided into three levels: (1) wearing appropriate personal protection, in a ventilated public place, and brief contact with a confirmed case ( $<15 \mathrm{~min})$, was considered to have a very low risk of infection; (2) close contact with the confirmed case (within $1 \mathrm{~m}$ ) but short contact ( $<15 \mathrm{~min}$ ) or long-distance contact in a ventilated public place $(>1 \mathrm{~m})$ but long contact time, or in a relatively closed environment with a confirmed case but no contact, was considered as medium risk of infection; (3) long (>15 min) close contact with a confirmed case within $1 \mathrm{~m}$, such as direct face-to-face contact, sharing the same ward, living in the same family, or traveling with the confirmed case, or moving together in the same neighborhood environment without any proper personal protection equipment, and medical staff treating the confirmed cases of COVID-19 without proper personal protection or illegal operation were considered to be at high risk of infection. In this study, 4 cases had come in contact with other suspected cases, but their risk of infection decreased with the elimination of diagnosis of the previously contacted suspected cases. Finally, they were reexamined by RT-PCR, and all were negative for SARS-CoV-2.

Cutting off the route of transmission is one of the three key links of epidemic prevention and control, emphasizing the importance of tracing the epidemiological history. For non-epidemic areas, there are four elements of epidemiological investigation within the latent period of the disease: (1) Whether there is sojourn history in the epidemic area. The epidemic area is determined by the situation at that time; previously, it was the domestic city of Wuhan. Recently, the focus has been transferred to foreign countries, such as the USA, Italy and Spain in Europe, Iran, and South Korea in Asia; (2) Whether there has been contact with confirmed cases. Then, the exposure risk can be classified according to the exposure risk level ${ }^{(16)}$; (3) Whether there was contact with suspected cases. The changes in diagnosis (confirmed or excluded) of the suspected case should be closely observed in the near future, and the risk level of infection of the case should be re-evaluated; (4) Whether there are clusters of disease. The occurrence of similar symptoms in $\geq 3$ cases is called aggregation. In this study, family aggregation was commonly observed while screening. Adherence to the basic strategies of disease: early detection, early diagnosis, early isolation, and early treatment.

CDVID-19 is mainly characterized by fever and respiratory symptoms, while digestive tract symptoms were rare, which is similar to the reports of early cases in Wuhan $(17,18)$. However, these symptoms are not typical, and other respiratory virus infections might occur, adding difficulty to the screening. In the present study, 2 asymptomatic confirmed CDVID-19 cases were detected, but no confirmed cases were found with only cough and no fever. Although these cases need to be focused on, additional cases need to be summarized. In this study, the number of confirmed patients with decreased WBC and increased CRP was significantly higher than that of the control group. These characteristics could be attributed to the systemic inflammatory response caused by SARS-CoV-2. Lym decline occurred in 50\% (4/8) of the confirmed COVID-19 cases. Considering that SARS-CoV-2 was similar to SARS-CoV ${ }^{(19)}$, it will attack human $T$ cells and lead to a decline in immune function. The presence of influenza A and B virus coinfection in confirmed cases should be assessed as a factor in aggravating the condition. Other 
respiratory virus infections might co-exist with COVID-19 in screening and should not be considered as an exclusion factor. In addition to the suspicion of COVID-19, other respiratory virus infections can be used as the differential diagnosis. The majority of the chest CT lesions of COVID-19 were bilateral diffuse or multifocal, followed by those in the left lower lung. The most common lesions were ground glass opacity in appearance. The lesions were often affecting the bilateral, subpleural lung parenchyma and progressing quickly, and the original lesions increased significantly after reexamination at an interval of 2 days (Fig. 2). Herein, no significant difference was detected in the pulmonary CT characteristics between confirmed cases and excluded suspected cases. Thus, we speculated that chest CT of viral pneumonia shared similar characteristics. The current study is a retrospective research, and hence, has some limitations. The sample size is relatively small, and the number of patients in the two groups is not equal, which may lead to data deviation. Therefore, large-sample studies are essential to provide an indepth understanding of COVID-19.

\section{Conclusion}

In conclusion, the use of only chest CT as the diagnostic criterion for COVID-19 was limited in nonepidemic areas. The diagnosis of infectious diseases should consider the detection of pathogens as the standard, focus on the epidemiology, combine with symptoms and signs, and follow the laboratory examination and comprehensive imaging assessment results to reduce misdiagnosis and missed diagnosis. The establishment of triage and screening strategies in hospitals, the control and isolation of suspected cases, and the treatment of confirmed cases at designated places can effectively cut off the transmission route. Strengthening the epidemic prevention and control and promoting the process of "triage-screening-isolation-transfer" for reference (Fig. 1) would aid in controlling the global epidemic.

\section{Declarations}

\section{Ethics approval and Consent to participate}

The study protocol was approved by the Ethics Committee of Southwest Hospital (document No.KY201989) and was carried out in accordance with the principles of the Declaration of Helsinki, 1964. Written informed consent was obtained from all the participants.

\section{Consent for publication}

We confirm that this manuscript has not been published elsewhere and is not under consideration in whole or in part by another journal. All authors have approved the manuscript and agree with submission to BMC Infectious Diseases.

\section{Availability of data and material}

Data regarding the SARS-CoV-2 (Severe acute respiratory syndrome coronavirus 2, 2019-nCoV) outbreak sequences can be found in GenBank/SRA, the NCBI Virus resource, and a specialized BLAST page that 
searches Betacoronavirus sequences.

\section{Competing interests}

The authors declare that they have no known competing financial interests or personal relationships that could have appeared to influence the work reported in this paper.

\section{Funding}

This work was sponsored by Mission Statement-A Construction Plan of Military Bio-Safety: Study on the integration of early warning, intervention and treatment of severe viral infectious diseases(17SAZ09); This work was partly supported by Chongqing Health Commission COVID-19 Project (2020NCPZX01)

\section{Authors' contributions}

All authors contributed to the study conception and design. Yan Zhu, Yan Guo collected the data; Juan Ma, Mao-shi Li, Yan Zhu analyzed and interpreted the data; Jia-fei Chen read the chest CT images; Yan Zhu, Yan Guo, Ming Liu provided patients to the study; Hui-min Liu, Ze-hui Yan, Guo-hong Deng designed the study; Huimin Liu wrote the manuscript; Qing Mao modified the manuscript.

\section{Acknowledgement}

The authors would like to thank Associate Chief Physician Jie Xia for the helpful suggestions, Matron Jian-ping You and Matron Sha Yang excellent technical assistance.

\section{Authors' information}

Not applicable

\section{Reference}

(1) Wuhan Health Commission informed about the current city pneumonia outbreak situation. http://wjw.wuhan.gov.cn/front/web/showDetail/2019123108989

(2) Wenjie Tan, Xiang Zhao, Xuejun Ma, Wenling Wang, Peihua Niu, Wenbo Xu, et al. Notes from the Field: A Novel Coronavirus Genome Identified in a Cluster of Pneumonia Cases - Wuhan, China 2019-2020 [J]. China CDC Weekly, 2020, 2(4): 61-62. doi: 10.46234/ccdcw2020.017

(3) Novel coronavirus-China. Disease outbreak news. http://www.who.int/csr/don/12-january-2020novel-coronavirus-china/en/. Update 12 January 2020.

(4) Coronaviridae Study Group of the International Committee on Taxonomy of Viruses. The species Severe acute respiratory syndrome-related coronavirus: classifying 2019-nCoV and naming it SARS-CoV2. Nat Microbiol. 2020;5(4):536-544. doi:10.1038/s41564-020-0695-z 
(5)Huang C, Wang Y, Li X, et al. Clinical features of patients infected with 2019 novel coronavirus in Wuhan, China. Lancet 2020; published online Jan 24. https://doi.org/10·1016/S0140- 6736(20)30183-5

(6) Li Q, Guan X, Wu P, et al. Early Transmission Dynamics in Wuhan, China, of Novel Coronavirus-Infected Pneumonia [published online ahead of print, 2020 Jan 29]. N Engl J Med.

2020;10.1056/NEJMoa2001316. doi:10.1056/NEJMoa2001316

(7) Riou J, Althaus CL. Pattern of early human-to-human transmission of Wuhan 2019 novel coronavirus (2019-nCoV), December 2019 to January 2020 [published correction appears in Euro Surveill. 2020 Feb;25(7):]. Euro Surveill. 2020;25(4):2000058. doi:10.2807/1560-7917.ES.2020.25.4.2000058

(8) WHO guidelines for the global surveillance of severe acute respiratory syndrome (SARS). https://www.who.int/csr/resources/publications/WHO_CDS_CSR_ARO_2004_1/en/.Updated recommendations, October 2004

(9) Middle East respiratory syndrome Case definition for reporting to WHO.

http://www.who.int/csr/disease/coronavirus_infections/case_definition/en/.Interim case definition, 26 July 2017.

(10) Backer JA, Klinkenberg D, Wallinga J. Incubation period of 2019 novel coronavirus (2019-nCoV) infections among travellers from Wuhan, China, 20-28 January 2020. Euro Surveill. 2020;25(5):2000062. doi:10.2807/1560-7917.ES.2020.25.5.2000062

(11) Wu JT, Leung K, Leung GM. Nowcasting and forecasting the potential domestic and international spread of the 2019-nCoV outbreak originating in Wuhan, China: a modelling study [published correction appears in Lancet. 2020 Feb 4;]. Lancet. 2020;395(10225):689-697. doi:10.1016/S0140-6736(20)302609

(12) Zhang J, Zhou L, Yang Y, Peng W, Wang W, Chen X. Therapeutic and triage strategies for 2019 novel coronavirus disease in fever clinics. Lancet Respir Med. 2020;8(3): e11-e12. doi:10.1016/S22132600(20)30071-0

(13) Jin YH, Cai L, Cheng ZS, et al. A rapid advice guideline for the diagnosis and treatment of 2019 novel coronavirus (2019-nCoV) infected pneumonia (standard version). Mil Med Res. 2020;7(1):4. Published 2020 Feb 6. doi:10.1186/s40779-020-0233-6

(14)de Groot RJ, Baker SC, Baric RS, et al. Middle East respiratory syndrome coronavirus (MERS-CoV): announcement of the Coronavirus Study Group. J Virol. 2013;87(14):7790-7792. doi:10.1128/JVI.0124413

(15) Zaki AM, van Boheemen S, Bestebroer TM, Osterhaus AD, Fouchier RA. Isolation of a novel coronavirus from a man with pneumonia in Saudi Arabia [published correction appears in N Engl J Med. 2013 Jul 25;369(4):394]. N Engl J Med. 2012;367(19):1814-1820. doi:10.1056/NEJMoa1211721 
(16) Bernard Stoecklin S, Rolland P, Silue Y, et al. First cases of coronavirus disease 2019 (COVID-19) in France: surveillance, investigations and control measures, January 2020. Euro Surveill. 2020;25(6):2000094. doi:10.2807/1560-7917.ES.2020.25.6.2000094

(17) Chen N, Zhou M, Dong X, et al. Epidemiological and clinical characteristics of 99 cases of 2019 novel coronavirus pneumonia in Wuhan, China: a descriptive study. Lancet. 2020;395(10223):507-513. doi:10.1016/S0140-6736(20)30211-7

(18) Shi H, Han X, Jiang N, et al. Radiological findings from 81 patients with COVID-19 pneumonia in Wuhan, China: a descriptive study [published online ahead of print, 2020 Feb 24]. Lancet Infect Dis. 2020; S1473-3099(20)30086-4. doi:10.1016/S1473-3099(20)30086-4

(19) Liu WJ, Zhao M, Liu K, et al. T-cell immunity of SARS-CoV: Implications for vaccine development against MERS-CoV. Antiviral Res. 2017; 137:82-92. doi: 10.1016/j.antiviral.2016.11.006

\section{Tables}

Table: 


\begin{tabular}{|c|c|c|c|c|}
\hline \multirow[b]{2}{*}{ Gender } & \multicolumn{4}{|c|}{ Total number $(n=38)$ Research group $(n=8)$ Control group $(n=30) P$ values } \\
\hline & & & & 0.426 \\
\hline Male & $19(50.0 \%)$ & $3(37.5 \%)$ & $16(53.3 \%)$ & 0.426 \\
\hline Female & $19(50.0 \%)$ & $5(62.5 \%)$ & $14(46.7 \%)$ & \\
\hline Age (years) & $39.1 \pm 14.5$ & $43.3 \pm 10.6$ & $38.0 \pm 15.3$ & 0.413 \\
\hline Time of onset (day) & $3.5(1.8-5.3)$ & $5.0(0.0-5.0)$ & $3.0(2.0-6.3)$ & 0.343 \\
\hline Length of stay (days) & $3.0(3.0-3.3)$ & $3.0(3.0-3.0)$ & $3.0(0.3-5.0)$ & 0.049 \\
\hline \multicolumn{5}{|l|}{ Epidemiological investigation } \\
\hline Return from Wuhan & $8(21.1 \%)$ & $4(50.0 \%)$ & $4(13.3 \%)$ & 0.024 \\
\hline \multicolumn{2}{|c|}{ A contact history of confirmed patients } & & & 0.011 \\
\hline Close contact & $6(15.8 \%)$ & $4(50.0 \%)$ & $2(6.7 \%)$ & \\
\hline Brief contact & $10(26.3 \%)$ & $1(12.5 \%)$ & $9(30.0 \%)$ & \\
\hline Clusters of disease & $12(31.65 \%)$ & $7(87.5 \%)$ & $5(16.7 \%)$ & $<0.001$ \\
\hline \multicolumn{2}{|c|}{ A contact history of suspected patients 4 (10.5\%) } & $0(0.0 \%)$ & $4(13.3 \%)$ & 0.275 \\
\hline \multicolumn{2}{|l|}{ The main symptoms } & & & 0.018 \\
\hline Fever (with or without cough) & $16(42.1 \%)$ & $3(37.5 \%)$ & $13(43.3 \%)$ & \\
\hline $37.3-38^{\circ} \mathrm{C}$ & $14(87.5 \%)$ & & & \\
\hline$>38^{\circ} \mathrm{C}$ & $2(15.0 \%)$ & & & \\
\hline Cough(without fever) & $11(28.9 \%)$ & $0(0.0 \%)$ & $11(36.7 \%)$ & \\
\hline Sore throat, itchy throat & $3(7.9 \%)$ & $0(0.0 \%)$ & $3(10.0 \%)$ & \\
\hline Fatigue, muscle aches, headaches & $3(7.9 \%)$ & $2(25.0 \%)$ & $1(3.3 \%)$ & \\
\hline Asymptomatic & $3(7.9 \%)$ & $2(25.0 \%)$ & $1(3.3 \%)$ & \\
\hline Difficulty in breathing & $1(2.6 \%)$ & $0(0.0 \%)$ & $1(3.3 \%)$ & \\
\hline Abdominal discomfort, diarrhea & $1(2.6 \%)$ & $1(12.5 \%)$ & $0(0.0 \%)$ & \\
\hline \multicolumn{2}{|l|}{ Other respiratory viruses } & & & 0.624 \\
\hline Influenza A & $7(18.4 \%)$ & $0(0.0 \%)$ & $7(23.3 \%)$ & \\
\hline Influenza B & $2(5.3 \%)$ & $1(12.5 \%)$ & $1(3.3 \%)$ & \\
\hline Influenza A + B & $5(13.2 \%)$ & $1(12.5 \%)$ & $4(13.3 \%)$ & \\
\hline Respiratory syncytial virus & $1(2.6 \%)$ & $0(0.0 \%)$ & $1(3.3 \%)$ & \\
\hline Adenovirus & $1(2.6 \%)$ & $0(0.0 \%)$ & $1(3.3 \%)$ & \\
\hline EBV & $1(2.6 \%)$ & $0(0.0 \%)$ & $1(3.3 \%)$ & \\
\hline No merger & $21(55.3 \%)$ & $6(75.0 \%)$ & $15(50.0 \%)$ & \\
\hline \multicolumn{5}{|l|}{ Biochemical index } \\
\hline $\mathrm{WBC}(10 \wedge 9 / \mathrm{L})$ & $7.2 \pm 2.8$ & $6.1 \pm 3.6$ & $7.4 \pm 2.5$ & 0.228 \\
\hline$<3.5$ & $3(7.9 \%)$ & $3(37.5 \%)$ & $0(0.0 \%)$ & $<0.001$ \\
\hline$\geq 3.5$ & $35(92.1 \%)$ & $5(62.5 \%)$ & $30(100.0 \%)$ & \\
\hline $\operatorname{Lym}(10 \wedge 9$ / L) & $1.1(0.9-1.9)$ & $1.0(0.7-1.2)$ & $1.3(0.9-2.1)$ & 0.082 \\
\hline$<1.1$ & $16(42.1 \%)$ & $4(50.0 \%)$ & $12(40.0 \%)$ & 0.611 \\
\hline$\geq 1.1$ & $22(57.9 \%)$ & $4(50.0 \%)$ & $18(60.0 \%)$ & \\
\hline Neu $\left(10^{\wedge} 9 / L\right)$ & $4.6(3.0-6.2)$ & $3.6(1.9-7.1)$ & $4.6(3.6-6.2)$ & 0.267 \\
\hline$<1.8$ & $1(2.6 \%)$ & $1(12.5 \%)$ & $0(0.0 \%)$ & 0.05 \\
\hline$\geq 1.8$ & $37(97.4 \%)$ & $7(87.5 \%)$ & $30(100.0 \%)$ & \\
\hline $\mathrm{ALT}(\mathrm{U} / \mathrm{L})$ & $30.3 \pm 19.9$ & $33.7 \pm 21.1$ & $29.3 \pm 19.8$ & 0.606 \\
\hline$\leq 42$ & $29(76.3 \%)$ & $5(62.5 \%)$ & $24(80.0 \%)$ & 0.301 \\
\hline$>42$ & $9(23.7 \%)$ & $3(37.5 \%)$ & $6(20.0 \%)$ & \\
\hline $\mathrm{Cr}(\mu \mathrm{mol} / \mathrm{L})$ & $60.1 \pm 19.6$ & $76.1 \pm 15.9$ & $55.9 \pm 18.4$ & 0.668 \\
\hline$\leq 97$ & $36(94.7 \%)$ & 7 (87.5\%) & $29(96.7 \%)$ & 0.302 \\
\hline$>97$ & $2(5.3 \%)$ & $1(12.5 \%)$ & $1(3.3 \%)$ & \\
\hline Glu (mmol/L) & $5.8(5.3-7.7)$ & $6.7(5.9-8.5)$ & $5.6(5.3-6.8)$ & 0.053 \\
\hline
\end{tabular}




\begin{tabular}{|c|c|c|c|c|}
\hline $3.9-6.1$ & $21(55.3 \%)$ & $2(25.0 \%)$ & $19(63.3 \%)$ & 0.053 \\
\hline$>6.1$ & $17(44.7 \%)$ & $6(75.0 \%)$ & $11(36.7 \%)$ & \\
\hline $\mathrm{LDH}(\mathrm{U} / \mathrm{L})$ & $193.4(171.7-253.4)$ & $212.5(176.0-311.8)$ & $\begin{array}{l}191.1 \\
(170.6-228.5)\end{array}$ & 0.316 \\
\hline $114-240$ & $28(73.7 \%)$ & $5(62.5 \%)$ & $23(76.7 \%)$ & 0.419 \\
\hline$>240$ & $10(26.3 \%)$ & $3(37.5 \%)$ & $7(23.3 \%)$ & \\
\hline CRP (mg/L) & $10.3(3.7-40.8)$ & $20.5(10.6-55.4)$ & $6.18(2.3-33.9)$ & 0.089 \\
\hline $0-8$ & $17(44.7 \%)$ & $1(12.5 \%)$ & $16(53.3)$ & 0.039 \\
\hline$>8$ & $21(55.3 \%)$ & $7(87.5 \%)$ & $14(46.7 \%)$ & \\
\hline PCT (ng/ml) & $0.04(0.01-0.75)$ & $0.65(0.0-0.16)$ & $0.03(0.01-0.06)$ & 0.187 \\
\hline $0-0.05$ & $25(65.8 \%)$ & $3(37.5 \%)$ & $22(73.3 \%)$ & 0.058 \\
\hline$>0.05$ & $13(34.2 \%)$ & $5(62.5 \%)$ & $8(26.7 \%)$ & \\
\hline \multicolumn{5}{|l|}{ Chest CT } \\
\hline Lesion & & & & 0.422 \\
\hline Bilateral diffuse or multifocal & $13(34.2 \%)$ & $5(62.5 \%)$ & $8(26.7 \%)$ & \\
\hline Right superior lung & $4(10.5 \%)$ & $0(0.0 \%)$ & $4(13.3 \%)$ & \\
\hline Right inferior lung & $3(7.9 \%)$ & $0(0.0 \%)$ & $3(10.0 \%)$ & \\
\hline Left superior lung & $2(5.3 \%)$ & $0(0.0 \%)$ & $2(6.7 \%)$ & \\
\hline Left inferior lung & $12(31.6 \%)$ & $2(25.0 \%)$ & $10(33.3 \%)$ & \\
\hline Both inferior lung & $4(10.5 \%)$ & $1(12.5 \%)$ & $3(10.0 \%)$ & \\
\hline Nature of the lesion & & & & 0.061 \\
\hline Ground-glass opacity & $22(57.9 \%)$ & $8(100.0 \%)$ & $14(46.7 \%)$ & \\
\hline Nodules & $8(21.1 \%)$ & $0(0.0 \%)$ & $8(26.7)$ & \\
\hline Fiber lesions & $2(5.3 \%)$ & $0(0.0 \%)$ & $2(6.7 \%)$ & \\
\hline Inflammatory lesions & $6(15.8 \%)$ & $0(0.0 \%)$ & $6(20.0 \%)$ & \\
\hline
\end{tabular}

\section{Figures}




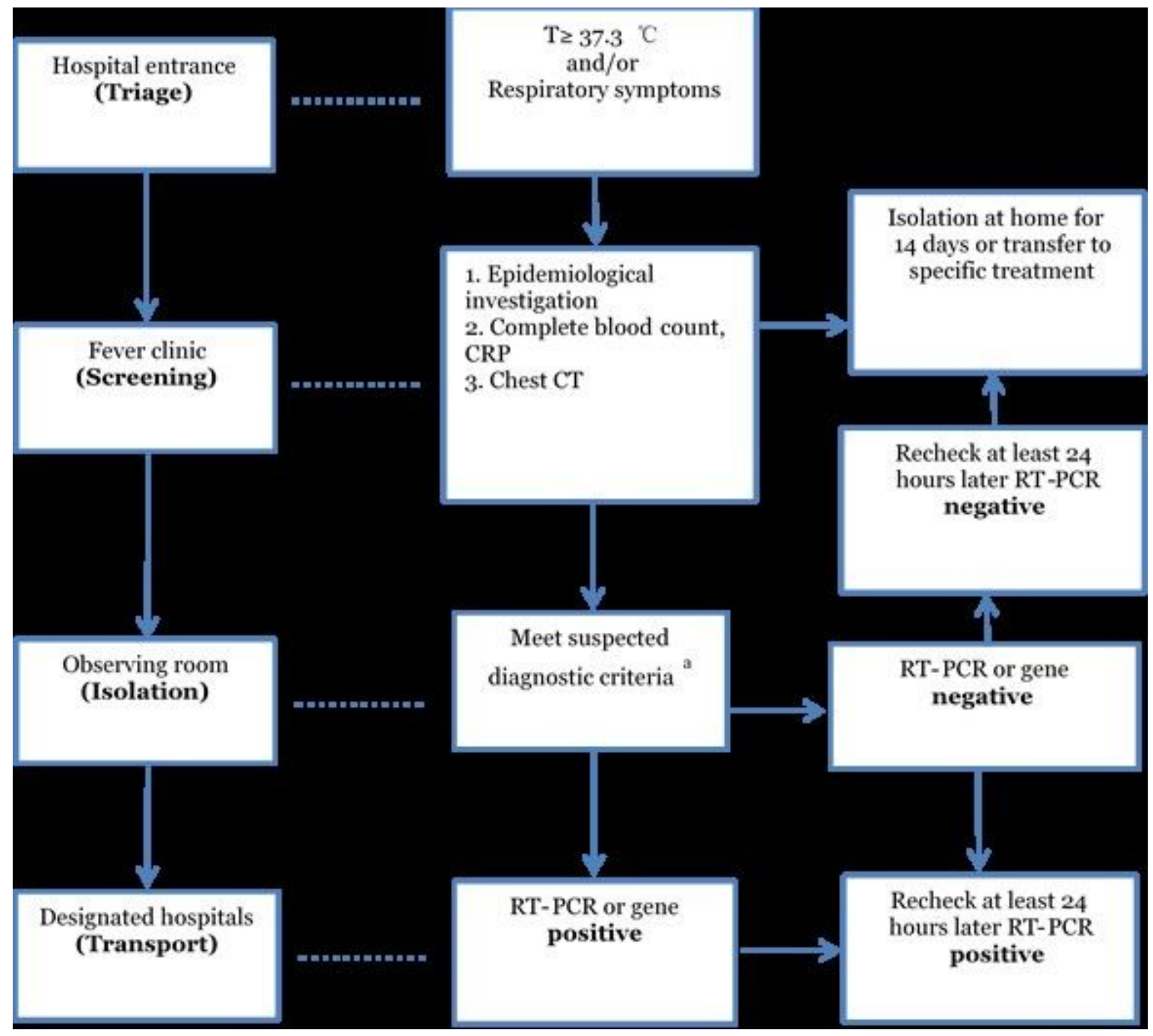

\section{Figure 1}

Figure 1 The process of "triage - screening - isolation - transport" a. Meet three clinical criteria, or two clinical criteria and one epidemiological criterion.
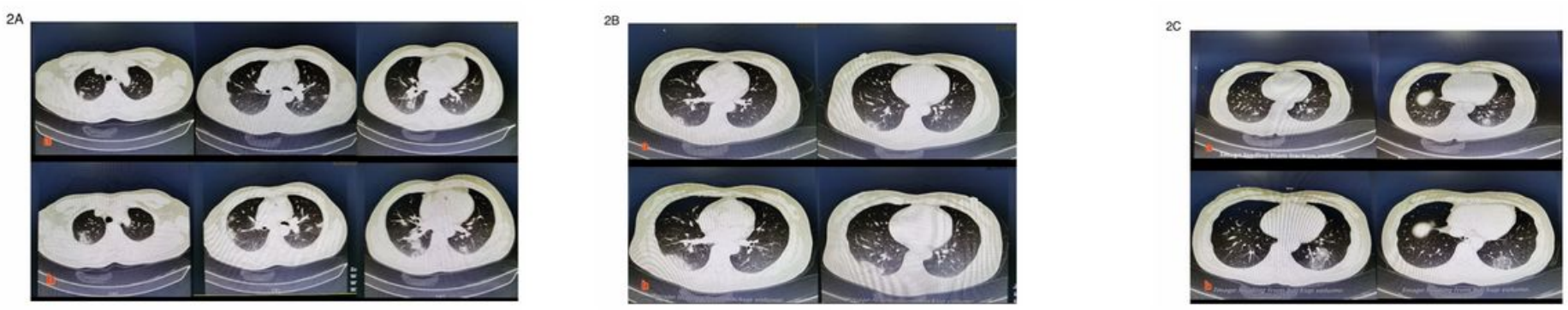

Figure 2

A囚 (a)Transverse chest CT imagesshowing bilateral diffuse and multifocal leisions on the day of admission (Jan 26th, 2020). (b) Transverse chest CT images from the same patient showing leisions

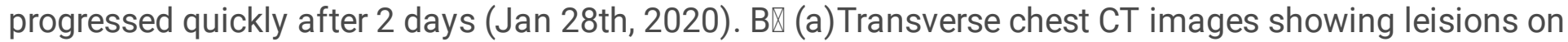
the left inferior lung on the day of admission (Jan 26th, 2020). (b) Transverse chest CT images from the 


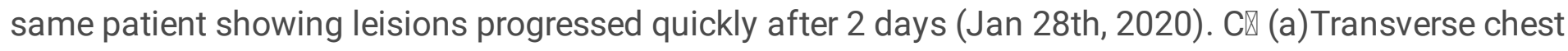
CT images showing leisions on the right inferior lung on the day of admission (Jan 26th, 2020). (b)

Transverse chest CT images from the same patient showing leisions progressed quickly after 2 days (Jan 28th, 2020). 